\title{
On positive solutions of second-order delayed differential system with indefinite weight
}

\author{
Fanglei Wang ${ }^{1 *}$ (D) and Ran Ding ${ }^{2}$
}

\section{"Correspondence:}

wang-fanglei@hotmail.com

'Department of Mathematics,

College of Science, Hohai University,

210098 Nanjing, P.R. China

Full list of author information is

available at the end of the article

\section{Springer}

\begin{abstract}
In this paper, we study the existence of positive solutions of a second-order delayed differential system, in which the weight functions may change sign. To prove our main results, we apply Krasnosel'skii's fixed point theorems in cones.
\end{abstract}

Keywords: Change of sign; Delay; Positive solutions; Fixed point theorem

\section{Introduction}

This paper is mainly concerned with the existence of positive solutions of a second-order two-delay differential system with

$$
\left\{\begin{array}{l}
x_{1}^{\prime \prime}(t)+h_{1}(t) f_{1}\left(x_{1}\left(t-\tau_{1}\right), x_{2}\left(t-\tau_{2}\right)\right)=0, \quad 0<t<1, \\
x_{2}^{\prime \prime}(t)+h_{2}(t) f_{2}\left(x_{1}\left(t-\tau_{1}\right), x_{2}\left(t-\tau_{2}\right)\right)=0, \quad 0<t<1, \\
x_{1}(t)=0, \quad-\tau_{1} \leq t \leq 0, \quad \text { and } \quad x_{1}(1)=0 \\
x_{2}(t)=0, \quad-\tau_{2} \leq t \leq 0, \quad \text { and } \quad x_{2}(1)=0
\end{array}\right.
$$

where $0<\tau_{1}<\tau_{2}<\frac{1}{2}$ are constants, and the weight functions $h_{i}(t)$ may change sign (see $\left.\left(\mathrm{H}_{1}\right)\right)$.

Many researchers have been attracted to study the theory, methodology and application of functional differential equations with delays, which have often been put forward as mathematical model to describe various natural phenomena [6,9]. One of the important aspects of research is that there are many papers devoted to studying nontrivial solutions of boundary-value problems for functional differential equations with delays. For example, J.W. Lee and D. O'Regan established the general existence principle of differentialdifference equations with delay type based on the nonlinear alternative (see [10,11]). Since then, T. Candan [4, 5] applied Krasnosel'skii's fixed point theorem for the sum of a completely continuous and a contraction mapping to prove the existence of positive periodic solutions for the first- (second-) order neutral differential equation. Y. Liu [12] applied the Mönch fixed point theorem to study the existence and uniqueness of solutions for the nonlinear functional differential equations on infinite interval. D. Bai and Y. Xu $[1,2]$

(c) The Author(s) 2021. This article is licensed under a Creative Commons Attribution 4.0 International License, which permits use sharing, adaptation, distribution and reproduction in any medium or format, as long as you give appropriate credit to the original author(s) and the source, provide a link to the Creative Commons licence, and indicate if changes were made. The images or other third party material in this article are included in the article's Creative Commons licence, unless indicated otherwise in a credit line to the material. If material is not included in the article's Creative Commons licence and your intended use is not permitted by statutory regulation or exceeds the permitted use, you will need to obtain permission directly from the copyright holder. To view a copy of this licence, visit http://creativecommons.org/licenses/by/4.0/. 
employed fixed-point theory to show how the parameters effect the number of positive solution for a two-delay singular differential system (1.1). In addition, to the best of our knowledge, many papers are concerned with the existence of positive solutions of differential equations with indefinite weight functions by using various methods, such as the fixed point theorems, the Leray-Schauder degree theory, bifurcation and so on. We refer the reader to $[3,7,13,14,16-18]$.

Motivated by the related references, in this paper we mainly apply the Krasnosel'skii fixed point theorems in cones to discuss the existence of positive solutions of problem (1.1), if the functions $h_{i}(t)$ and $f_{i}\left(x_{1}, x_{2}\right)$ satisfy the following assumptions:

$\left(\mathrm{H}_{1}\right) h_{i}:[0,1] \rightarrow(-\infty,+\infty)$ are continuous, and there exists a $\xi \in\left(0,1-\tau_{2}\right)$ such that

$$
\begin{cases}h_{i}(t) \geq 0, & \text { if } t \in[0, \xi], \\ h_{i}(t) \equiv 0, & \text { if } t \in\left[\xi, \xi+\tau_{2}\right], \\ h_{i}(t) \leq 0, & \text { if } t \in\left[\xi+\tau_{2}, 1\right] .\end{cases}
$$

Furthermore, $h_{i}$ do not vanish identically on any subinterval of $[0, \xi]$ and $\left[\xi+\tau_{2}, 1\right]$.

$\left(\mathrm{H}_{2}\right) f_{i}: \mathbb{R}^{+2} \rightarrow \mathbb{R}^{+}$are continuous and there exists a $\theta \in(0,1]$ such that

$$
\begin{gathered}
f_{i}(u, v) \geq \theta \phi_{i}(u, v), \\
\phi_{i}(u, v)=\max \left\{f_{i}\left(x_{1}, x_{2}\right): 0 \leq x_{1} \leq u, 0 \leq x_{2} \leq v\right\} .
\end{gathered}
$$

$\left(\mathrm{H}_{3}\right)$ There exist positive constants $k_{i}, \alpha_{i}$ and continuous functions

$$
F_{i}\left(x_{1}, x_{2}\right): \mathbb{R}^{+2} \rightarrow \mathbb{R}^{+}
$$

satisfying

(i) $F_{i}\left(x_{1}, x_{2}\right)$ are strictly increasing functions with respective to $\left(x_{1}, x_{2}\right)$,

(ii) $F_{i}\left(\lambda x_{1}, \lambda x_{2}\right)=\lambda^{\alpha_{i}} F_{i}\left(x_{1}, x_{2}\right)$,

such that

$$
k_{i} F_{i}\left(x_{1}, x_{2}\right) \leq f_{i}\left(x_{1}, x_{2}\right) \leq F_{i}\left(x_{1}, x_{2}\right), \quad\left(x_{1}, x_{2}\right) \in \mathbb{R}^{+2} .
$$

$\left(\mathrm{H}_{4}\right)$ There exist $\sigma_{i}$ satisfying $\tau_{2}<\sigma_{i}<\xi$ such that

$$
\theta^{2} k_{i}\left(\sigma_{i}-\tau_{2}\right)^{\alpha_{i}} \int_{\sigma_{i}}^{\xi} G(t, s) h_{i}^{+}(s) d s \geq \xi^{\alpha_{i}} \int_{\xi+\tau_{2}}^{1} G(t, s) h_{i}^{-}(s) d s .
$$

\section{Preliminaries}

Let

$$
E_{i}=\left\{x_{i} \in C\left[-\tau_{i}, 1\right]: x_{i}(t)=0, \forall t \in\left[-\tau_{i}, 0\right] \text { and } x_{i}(1)=0\right\} \quad(i=1,2)
$$

be a Banach space with norm $\left|x_{i}(t)\right|_{i}=\max _{-\tau_{i} \leq t \leq 1} x_{i}(t)=\max _{0 \leq t \leq 1} x_{i}(t)$. Then we can define a Banach space $E$ by $E_{1} \times E_{2}$ with norm $\|x\|=\max \left\{\left|x_{1}\right|_{1},\left|x_{2}\right|_{2}\right\}$, for $x=\left(x_{1}, x_{2}\right) \in E$. Also, define a subcone $K \subset E$ by

$$
K=\left\{x \in E: x(t) \geq 0, x_{i} \text { is concave on }[0, \xi] \text { and convex on }[\xi, 1]\right\} .
$$


For $\forall x(t) \in K$, it is obvious that

$$
\left|x_{i}\right|_{i}=\max _{0 \leq t \leq \xi} x_{i}(t)
$$

For any $\gamma>0$, in the following paragraphs, we set

$$
K_{\gamma}=\{x \in K:\|x\|<\gamma\}
$$

and

$$
\partial K_{\gamma}=\{x \in K:\|x\|=\gamma\} .
$$

Define an operator $T$ by $T x(t)=\left(T_{1} x(t), T_{2} x(t)\right)$, where

$$
T_{i} x(t)= \begin{cases}\int_{0}^{1} G(t, s) h_{i}(s) f_{i}\left(x_{1}\left(s-\tau_{1}\right), x_{2}\left(s-\tau_{2}\right)\right) d s, & 0<t \leq 1 \\ 0, & -\tau_{i} \leq t \leq 0\end{cases}
$$

where

$$
G(t, s)= \begin{cases}(1-t) s, & 0 \leq s \leq t \leq 1 \\ t(1-s), & 0 \leq t \leq s \leq 1\end{cases}
$$

Now solutions of (1.1) can be rewritten as fixed points of $T$ in Banach space $E$.

Lemma 2.1 Assume that $\left(\mathrm{H}_{1}\right)-\left(\mathrm{H}_{4}\right)$ hold. Then the operator $T$ is positive and $T: K \rightarrow K$ is completely continuous.

Proof First of all, we show that the operator $T_{i}$ is positive, namely, for any $x(t) \in K$, we have

$$
\int_{0}^{1} G(t, s) h_{i}(s) f_{i}\left(x_{1}\left(s-\tau_{1}\right), x_{2}\left(s-\tau_{2}\right)\right) d s \geq \int_{0}^{\sigma_{i}} G(t, s) h_{i}^{+}(s) f_{i}\left(x_{1}\left(s-\tau_{1}\right), x_{2}\left(s-\tau_{2}\right)\right) d s
$$

For any $x(t) \in K$, we can obtain

$$
x_{i}(t) \geq q(t) x_{i}(\xi), \quad t \in[0, \xi] \quad \text { and } \quad x_{i}(t) \leq q(t) x_{i}(\xi), \quad t \in[\xi, 1]
$$

where $q(t):[0,1] \rightarrow[0,1]$ defined by $q(t)=\min \left\{\frac{t}{\xi}, \frac{1-t}{1-\xi}\right\}$. Hence, we have

$$
\min _{s \in\left[\sigma_{1}, \xi\right]} q\left(s-\tau_{i}\right)=\frac{\sigma_{1}-\tau_{i}}{\xi}, \quad \max _{s \in\left[\xi+\tau_{2}, 1\right]} q\left(s-\tau_{i}\right)=\frac{1-\xi-\tau_{2}+\tau_{i}}{1-\xi} .
$$

Then, from $\left(\mathrm{H}_{1}\right)-\left(\mathrm{H}_{4}\right)$, it follows that

$$
\begin{aligned}
& \int_{0}^{1} G(t, s) h_{1}(s) f_{1}\left(x_{1}\left(s-\tau_{1}\right), x_{2}\left(s-\tau_{2}\right)\right) d s-\int_{0}^{\sigma_{1}} G(t, s) h_{1}^{+}(s) f_{1}\left(x_{1}\left(s-\tau_{1}\right), x_{2}\left(s-\tau_{2}\right)\right) d s \\
& \quad=\int_{\sigma_{1}}^{\xi} G(t, s) h_{1}^{+}(s) f_{1}\left(x_{1}\left(s-\tau_{1}\right), x_{2}\left(s-\tau_{2}\right)\right) d s
\end{aligned}
$$




$$
\begin{aligned}
& -\int_{\xi+\tau_{2}}^{1} G(t, s) h_{1}^{-}(s) f_{1}\left(x_{1}\left(s-\tau_{1}\right), x_{2}\left(s-\tau_{2}\right)\right) d s \\
& \geq \theta \int_{\sigma_{1}}^{\xi} G(t, s) h_{1}^{+}(s) \phi_{1}\left(x_{1}\left(s-\tau_{1}\right), x_{2}\left(s-\tau_{2}\right)\right) d s \\
& -\int_{\xi+\tau_{2}}^{1} G(t, s) h_{1}^{-}(s) \phi_{1}\left(x_{1}\left(s-\tau_{1}\right), x_{2}\left(s-\tau_{2}\right)\right) d s \\
& \geq \theta \int_{\sigma_{1}}^{\xi} G(t, s) h_{1}^{+}(s) \phi_{1}\left(q\left(s-\tau_{1}\right) x_{1}(\xi), q\left(s-\tau_{2}\right) x_{2}(\xi)\right) d s \\
& -\int_{\xi+\tau_{2}}^{1} G(t, s) h_{1}^{-}(s) \phi_{1}\left(q\left(s-\tau_{1}\right) x_{1}(\xi), q\left(s-\tau_{2}\right) x_{2}(\xi)\right) d s \\
& \geq \theta \int_{\sigma_{1}}^{\xi} G(t, s) h_{1}^{+}(s) f_{1}\left(q\left(s-\tau_{1}\right) x_{1}(\xi), q\left(s-\tau_{2}\right) x_{2}(\xi)\right) d s \\
& -\frac{1}{\theta} \int_{\xi+\tau_{2}}^{1} G(t, s) h_{1}^{-}(s) f_{1}\left(q\left(s-\tau_{1}\right) x_{1}(\xi), q\left(s-\tau_{2}\right) x_{2}(\xi)\right) d s \\
& =\frac{1}{\theta}\left[\theta^{2} \int_{\sigma_{1}}^{\xi} G(t, s) h_{1}^{+}(s) f_{1}\left(q\left(s-\tau_{1}\right) x_{1}(\xi), q\left(s-\tau_{2}\right) x_{2}(\xi)\right) d s\right. \\
& \left.-\int_{\xi+\tau_{2}}^{1} G(t, s) h_{1}^{-}(s) f_{1}\left(q\left(s-\tau_{1}\right) x_{1}(\xi), q\left(s-\tau_{2}\right) x_{2}(\xi)\right) d s\right] \\
& \geq \frac{1}{\theta}\left[\theta^{2} \int_{\sigma_{1}}^{\xi} G(t, s) h_{1}^{+}(s) k_{1} F_{1}\left(q\left(s-\tau_{1}\right) x_{1}(\xi), q\left(s-\tau_{2}\right) x_{2}(\xi)\right) d s\right. \\
& \left.-\int_{\xi+\tau_{2}}^{1} G(t, s) h_{1}^{-}(s) F_{1}\left(q\left(s-\tau_{1}\right) x_{1}(\xi), q\left(s-\tau_{2}\right) x_{2}(\xi)\right) d s\right] \\
& \geq \frac{1}{\theta}\left[\theta^{2} \int_{\sigma_{1}}^{\xi} G(t, s) h_{1}^{+}(s) k_{1} F_{1}\left(\min _{\left[\sigma_{1}, \xi\right]} q\left(s-\tau_{1}\right) x_{1}(\xi), \min _{\left[\sigma_{1}, \xi\right]} q\left(s-\tau_{2}\right) x_{2}(\xi)\right) d s\right. \\
& \left.-\int_{\xi+\tau_{2}}^{1} G(t, s) h_{1}^{-}(s) F_{1}\left(\max _{\left[\xi+\tau_{2}, 1\right]} q\left(s-\tau_{1}\right) x_{1}(\xi), \max _{\left[\xi+\tau_{2}, 1\right]} q\left(s-\tau_{2}\right) x_{2}(\xi)\right) d s\right] \\
& =\frac{1}{\theta}\left[\theta^{2} \int_{\sigma_{1}}^{\xi} G(t, s) h_{1}^{+}(s) \frac{k_{1}}{\xi^{\alpha_{1}}} F_{1}\left(\left(\sigma_{1}-\tau_{1}\right) x_{1}(\xi),\left(\sigma_{1}-\tau_{2}\right) x_{2}(\xi)\right) d s\right. \\
& \left.-\int_{\xi+\tau_{2}}^{1} G(t, s) h_{1}^{-}(s) \frac{1}{(1-\xi)^{\alpha_{1}}} F_{1}\left(\left(1-\xi-\tau_{2}+\tau_{1}\right) x_{1}(\xi),(1-\xi) x_{2}(\xi)\right) d s\right] \\
& \geq \frac{1}{\theta}\left[\theta^{2} \int_{\sigma_{1}}^{\xi} G(t, s) h_{1}^{+}(s) \frac{k_{1}}{\xi^{\alpha_{1}}} F_{1}\left(\left(\sigma_{1}-\tau_{2}\right) x_{1}(\xi),\left(\sigma_{1}-\tau_{2}\right) x_{2}(\xi)\right) d s\right. \\
& \left.-\int_{\xi+\tau_{2}}^{1} G(t, s) h_{1}^{-}(s) \frac{1}{(1-\xi)^{\alpha_{1}}} F_{1}\left((1-\xi) x_{1}(\xi),(1-\xi) x_{2}(\xi)\right) d s\right] \\
& =\frac{1}{\theta}\left[\theta^{2} \int_{\sigma_{1}}^{\xi} G(t, s) h_{1}^{+}(s) \frac{k_{1}}{\xi^{\alpha_{1}}}\left(\sigma_{1}-\tau_{2}\right)^{\alpha_{1}} d s\right. \\
& \left.-\int_{\xi+\tau_{2}}^{1} G(t, s) h_{1}^{-}(s) \frac{1}{(1-\xi)^{\alpha_{1}}}(1-\xi)^{\alpha_{1}} d s\right] \cdot F_{1}\left(x_{1}(\xi), x_{2}(\xi)\right) \\
& \geq 0 \text {, }
\end{aligned}
$$

which implies that the operator $T_{1}$ is positive. 
In the similar way, we also get

$$
\int_{0}^{1} G(t, s) h_{2}(s) f_{2}\left(x_{1}\left(s-\tau_{1}\right), x_{2}\left(s-\tau_{2}\right)\right) d s \geq \int_{0}^{\sigma_{2}} G(t, s) h_{2}^{+}(s) f_{2}\left(x_{1}\left(s-\tau_{1}\right), x_{2}\left(s-\tau_{2}\right)\right) d s,
$$

which implies that the operator $T_{2}$ is positive.

Secondly, from the conditions $\left(\mathrm{H}_{1}\right)-\left(\mathrm{H}_{3}\right)$, we can obtain

$$
\left(T_{i} x\right)^{\prime \prime}(t) \leq 0, \quad t \in[0, \xi] \quad \text { and } \quad\left(T_{i} x\right)^{\prime \prime}(t) \geq 0, \quad t \in[\xi, 1]
$$

Ultimately, from the standard process, we can prove that $T: K \rightarrow K$ is completely continuous.

The proofs of this paper are mainly based on the Krasnosel'skii fixed point theorems in cones such as the following.

Lemma 2.2 ([8]) Let E be a Banach space, and $K \subset E$ be a cone in E. Assume that $\Omega_{1}, \Omega_{2}$ are open subsets of $E$ with $0 \in \Omega_{1}, \bar{\Omega}_{1} \subset \Omega_{2}$, and let $T: K \cap\left(\bar{\Omega}_{2} \backslash \Omega_{1}\right) \rightarrow K$ be a completely continuous operator such that either

(i) $\|T u\| \leq\|u\|, u \in K \cap \partial \Omega_{1}$ and $\|T u\| \geq\|u\|, u \in K \cap \partial \Omega_{2}$; or

(ii) $\|T u\| \geq\|u\|, u \in K \cap \partial \Omega_{1}$ and $\|T u\| \leq\|u\|, u \in K \cap \partial \Omega_{2}$.

Then $T$ has a fixed point in $K \cap\left(\bar{\Omega}_{2} \backslash \Omega_{1}\right)$.

Lemma $2.3([15])$ Let $(X,|\cdot|)$ be a normal linear space; $K_{1}, K_{2} \subset X$ two cones; $K: K_{1} \times K_{2}$; $r, R \in R_{+}^{2}$ with $0<r_{i}<R_{i}$ for $i=1,2, K_{r, R}:=\left\{u=\left(u_{1}, u_{2}\right) \in K: r_{i} \leq\left|u_{i}\right| \leq R_{i}, i=1,2\right\}$, and let $T=\left(T_{1}, T_{2}\right)$ be a compact map. Assume that, for each $i \in\{1,2\}$, one of the following conditions is satisfied in $K_{r, R}$ :

(a) $T_{i}(u) \nless(\geq) u_{i}$ if $\left|u_{i}\right|=r_{i}$, and $T_{i}(u) \ngtr(\leq) u_{i}$ if $\left|u_{i}\right|=R_{i}$;

(b) $T_{i}(u) \ngtr(\leq) u_{i}$ if $\left|u_{i}\right|=r_{i}$, and $T_{i}(u) \nless(\geq) u_{i}$ if $\left|u_{i}\right|=R_{i}$.

Then $T$ has a fixed point $u$ in $K$ with $r_{i} \leq\left|u_{i}\right| \leq R_{i}$.

\section{Main results I}

Define a function $\delta(t)$ by

$$
\delta(t)=\min \left\{\frac{t}{\xi}, \frac{\xi-t}{\xi}\right\}, \quad t \in[0, \xi] .
$$

Then, for $i=1,2$, let

$$
\begin{aligned}
& \gamma_{i}=\min \left\{\min _{\frac{\sigma_{i}+\tau_{2}}{2} \leq s \leq \sigma_{i}} \delta\left(s-\tau_{1}\right), \min _{\frac{\sigma_{i}+\tau_{2}}{2} \leq s \leq \sigma_{i}} \delta\left(s-\tau_{2}\right)\right\}, \\
& A_{i}=k_{i} \gamma_{i}^{\alpha_{i}} \max _{t \in[0, \xi]} G(t, t) \cdot \int_{\frac{\sigma_{i}+\tau_{2}}{2}}^{\sigma_{i}} G(s, s) h_{i}^{+}(s) d s, \\
& B_{i}=\int_{\tau_{1}}^{\tau_{2}} G(s, s) h_{i}^{+}(s) d s, C_{i}=\int_{\tau_{2}}^{\xi} G(s, s) h_{i}^{+}(s) d s .
\end{aligned}
$$


Theorem 3.1 Assume that $\left(\mathrm{H}_{1}\right)-\left(\mathrm{H}_{4}\right)$ hold. If there exist two positive constants $r, R$ with $r<R$, satisfying

$$
\begin{aligned}
& \min \left\{A_{1} F_{1}(r, 0), A_{2} F_{2}(0, r)\right\} \geq r, \\
& \max \left\{B_{1} F_{1}(R, 0)+C_{1} F_{1}(R, R), B_{2} F_{2}(R, 0)+C_{2} F_{2}(R, R)\right\} \leq R,
\end{aligned}
$$

then problem (1.1) at least has a positive solution.

Proof On one hand, for any $x \in \partial K_{R}$, we have $0 \leq x_{1}, x_{2} \leq R$. Then, by the assumptions, we have

$$
\begin{aligned}
\left|T_{1} x\right|_{1} \leq & \int_{0}^{1} G(s, s) h_{1}(s) f_{1}\left(x_{1}\left(s-\tau_{1}\right), x_{2}\left(s-\tau_{2}\right)\right) d s \\
= & \int_{0}^{\xi} G(s, s) h_{1}^{+}(s) f_{1}\left(x_{1}\left(s-\tau_{1}\right), x_{2}\left(s-\tau_{2}\right)\right) d s \\
& -\int_{\xi}^{1} G(s, s) h_{1}^{-}(s) f_{1}\left(x_{1}\left(s-\tau_{1}\right), x_{2}\left(s-\tau_{2}\right)\right) d s \\
\leq & \int_{0}^{\xi} G(s, s) h_{1}^{+}(s) f_{1}\left(x_{1}\left(s-\tau_{1}\right), x_{2}\left(s-\tau_{2}\right)\right) d s \\
= & \left(\int_{\tau_{1}}^{\tau_{2}}+\int_{\tau_{2}}^{\xi}\right) G(s, s) h_{1}^{+}(s) f_{1}\left(x_{1}\left(s-\tau_{1}\right), x_{2}\left(s-\tau_{2}\right)\right) d s \\
\leq & \int_{\tau_{1}}^{\tau_{2}} G(s, s) h_{1}^{+}(s) f_{1}\left(x_{1}\left(s-\tau_{1}\right), 0\right) d s \\
& +\int_{\tau_{2}}^{\xi} G(s, s) h_{1}^{+}(s) f_{1}\left(x_{1}\left(s-\tau_{1}\right), x_{2}\left(s-\tau_{2}\right)\right) d s \\
\leq & \int_{\tau_{1}}^{\tau_{2}} G(s, s) h_{1}^{+}(s) F_{1}\left(x_{1}\left(s-\tau_{1}\right), 0\right) d s \\
& +\int_{\tau_{2}}^{\xi} G(s, s) h_{1}^{+}(s) F_{1}\left(x_{1}\left(s-\tau_{1}\right), x_{2}\left(s-\tau_{2}\right)\right) d s \\
\leq & \int_{\tau_{1}}^{\tau_{2}} G(s, s) h_{1}^{+}(s) F_{1}(R, 0) d s \\
& +\int_{\tau_{2}}^{\xi} G(s, s) h_{1}^{+}(s) F_{1}(R, R) d s \\
\leq & R .
\end{aligned}
$$

In the similar way, we also have

$$
\begin{aligned}
\left|T_{2} x\right|_{2} & \leq \int_{0}^{1} G(s, s) h_{2}(s) f_{2}\left(x_{1}\left(s-\tau_{1}\right), x_{2}\left(s-\tau_{2}\right)\right) d s \\
& \leq \int_{\tau_{1}}^{\tau_{2}} G(s, s) h_{2}^{+}(s) F_{2}(R, 0) d s+\int_{\tau_{2}}^{\xi} G(s, s) h_{2}^{+}(s) F_{2}(R, R) d s \\
& \leq R .
\end{aligned}
$$


So, from the above discussions, we get

$$
\|T x\|=\max \left\{\left|T_{1} x\right|_{1},\left|T_{2} x\right|_{2}\right\} \leq R=\|x\|, \quad \text { for } x \in \partial K_{R} .
$$

On the other hand, for any $x(t) \in K$, from the concave property of $x_{i}(t)$ it follows that

$$
x_{i}(t) \geq \delta(t)\left|x_{i}(t)\right|_{i}, \quad t \in[0, \xi]
$$

Then, for any $x(t) \in \partial K_{r}$, we have the following.

Case I: if $\left|x_{1}(t)\right|_{1}=r$, then $\delta(t) r \leq x_{1}(t) \leq r$ and $0 \leq x_{2}(t) \leq r$. By $\left(\mathrm{H}_{2}\right)$, we have

$$
\begin{aligned}
& \max _{t \in[0, \xi]} T_{1} x(t)=\max _{t \in[0, \xi]} \int_{0}^{1} G(t, s) h_{1}(s) f_{1}\left(x_{1}\left(s-\tau_{1}\right), x_{2}\left(s-\tau_{2}\right)\right) d s \\
& \geq \max _{t \in[0, \xi]} \int_{0}^{\sigma_{1}} G(t, s) h_{1}^{+}(s) f_{1}\left(x_{1}\left(s-\tau_{1}\right), x_{2}\left(s-\tau_{2}\right)\right) d s \\
& \geq \max _{t \in[0, \xi]} G(t, t) \cdot \int_{0}^{\sigma_{1}} G(s, s) h_{1}^{+}(s) f_{1}\left(x_{1}\left(s-\tau_{1}\right), x_{2}\left(s-\tau_{2}\right)\right) d s \\
& \geq k_{1} \max _{t \in[0, \xi]} G(t, t) \cdot \int_{\frac{\sigma_{1}+\tau_{2}}{2}}^{\sigma_{1}} G(s, s) h_{1}^{+}(s) F_{1}\left(x_{1}\left(s-\tau_{1}\right), x_{2}\left(s-\tau_{2}\right)\right) d s \\
& \geq k_{1} \max _{t \in[0, \xi]} G(t, t) \cdot \int_{\frac{\sigma_{1}+\tau_{2}}{2}}^{\sigma_{1}} G(s, s) h_{1}^{+}(s) F_{1}\left(\delta\left(s-\tau_{1}\right)\left|x_{1}\right|_{1}, \delta\left(s-\tau_{2}\right)\left|x_{2}\right|_{2}\right) d s \\
& \geq k_{1} \max _{t \in[0, \xi]} G(t, t) \cdot \int_{\frac{\sigma_{1}+\tau_{2}}{2}}^{\sigma_{1}} G(s, s) h_{1}^{+}(s) F_{1}\left(\gamma_{1}\left|x_{1}\right|_{1}, \gamma_{1}\left|x_{2}\right|_{2}\right) d s \\
& =k_{1} \gamma_{1}^{\alpha_{1}} \max _{t \in[0, \xi]} G(t, t) \cdot \int_{\frac{\sigma_{1}+\tau_{2}}{2}}^{\sigma_{1}} G(s, s) h_{1}^{+}(s) F_{1}\left(\left|x_{1}\right|_{1},\left|x_{2}\right|_{2}\right) d s \\
& \geq k_{1} \gamma_{1}^{\alpha_{1}} \max _{t \in[0, \xi]} G(t, t) \cdot \int_{\frac{\sigma_{1}+\tau_{2}}{2}}^{\sigma_{1}} G(s, s) h_{1}^{+}(s) F_{1}(r, 0) d s \\
& =k_{1} \gamma_{1}^{\alpha_{1}} \max _{t \in[0, \xi]} G(t, t) \cdot \int_{\frac{\sigma_{1}+\tau_{2}}{2}}^{\sigma_{1}} G(s, s) h_{1}^{+}(s) d s \cdot F_{1}(r, 0) \\
& \geq r \text {. }
\end{aligned}
$$

Case II: if $\left|x_{1}(t)\right|_{1}<r$, then $0 \leq x_{1}(t)<r$ and $\delta(t) r \leq x_{2}(t) \leq r$. By $\left(\mathrm{H}_{2}\right)$, we have

$$
\begin{aligned}
\max _{t \in[0, \xi]} T_{2} x(t) & =\max _{t \in[0, \xi]} \int_{0}^{1} G(t, s) h_{2}(s) f_{2}\left(x_{1}\left(s-\tau_{1}\right), x_{2}\left(s-\tau_{2}\right)\right) d s \\
& \geq \max _{t \in[0, \xi]} \int_{0}^{\sigma_{2}} G(t, s) h_{2}^{+}(s) f_{2}\left(x_{1}\left(s-\tau_{1}\right), x_{2}\left(s-\tau_{2}\right)\right) d s \\
& \geq \max _{t \in[0, \xi]} G(t, t) \cdot \int_{0}^{\sigma_{2}} G(s, s) h_{2}^{+}(s) f_{2}\left(x_{1}\left(s-\tau_{1}\right), x_{2}\left(s-\tau_{2}\right)\right) d s \\
& \geq k_{2} \max _{t \in[0, \xi]} G(t, t) \cdot \int_{\frac{\sigma_{2}+\tau_{2}}{2}}^{\sigma_{2}} G(s, s) h_{2}^{+}(s) F_{2}\left(x_{1}\left(s-\tau_{1}\right), x_{2}\left(s-\tau_{2}\right)\right) d s \\
& \geq k_{2} \max _{t \in[0, \xi]} G(t, t) \cdot \int_{\frac{\sigma_{2}+\tau_{2}}{2}}^{\sigma_{2}} G(s, s) h_{1}^{+}(s) F_{2}\left(\delta\left(s-\tau_{1}\right)\left|x_{1}\right|_{1}, \delta\left(s-\tau_{2}\right)\left|x_{2}\right|_{2}\right) d s \\
& \geq k_{2} \max _{t \in[0, \xi]} G(t, t) \cdot \int_{\frac{\sigma_{2}+\tau_{2}}{2}}^{\sigma_{2}} G(s, s) h_{1}^{+}(s) F_{2}\left(\gamma_{2}\left|x_{1}\right|_{1}, \gamma_{2}\left|x_{2}\right|_{2}\right) d s
\end{aligned}
$$




$$
\begin{aligned}
& =k_{2} \gamma_{2}^{\alpha_{2}} \max _{t \in[0, \xi]} G(t, t) \cdot \int_{\frac{\sigma_{2}+\tau_{2}}{2}}^{\sigma_{2}} G(s, s) h_{2}^{+}(s) F_{2}\left(\left|x_{1}\right|_{1},\left|x_{2}\right|_{2}\right) d s \\
& \geq k_{2} \gamma_{2}^{\alpha_{2}} \max _{t \in[0, \xi]} G(t, t) \cdot \int_{\frac{\sigma_{2}+\tau_{2}}{2}}^{\sigma_{2}} G(s, s) h_{2}^{+}(s) F_{2}(0, r) d s \\
& =k_{2} \gamma_{2}^{\alpha_{2}} \max _{t \in[0, \xi]} G(t, t) \cdot \int_{\frac{\sigma_{2}+\tau_{2}}{2}}^{\sigma_{2}} G(s, s) h_{2}^{+}(s) d s \cdot F_{2}(0, r) \\
& \geq r .
\end{aligned}
$$

So, from the above discussions, we get

$$
\|T x\|=\max \left\{\left|T_{1} x\right|_{1},\left|T_{2} x\right|_{2}\right\} \geq r=\|x\|, \quad \text { for } x \in \partial K_{r} .
$$

Therefore, from (3.1) and (3.2) and Lemma 2.2, the operator $T$ has a fixed point in $K \cap$ $\left(\bar{\Omega}_{R} \backslash \Omega_{r}\right)$.

As by a similar proof to Theorem 3.1, we also get the following.

Theorem 3.2 Assume that $\left(\mathrm{H}_{1}\right)-\left(\mathrm{H}_{4}\right)$ hold. If there exist two positive constants $r, R$ with $r<R$, satisfying

$$
\begin{aligned}
& \min \left\{A_{1} F_{1}(R, 0), A_{2} F_{2}(0, R)\right\} \geq R, \\
& \max \left\{B_{1} F_{1}(r, 0)+C_{1} F_{1}(r, r), B_{2} F_{2}(r, 0)+C_{2} F_{2}(r, r)\right\} \leq r,
\end{aligned}
$$

then problem (1.1) at least has a positive solution.

\section{Main results II}

Theorem 4.1 Assume that $\left(\mathrm{H}_{1}\right)-\left(\mathrm{H}_{4}\right)$ hold. If there exist four positive constants $r_{1}, r_{2}, R_{1}$, $R_{2}$ with $r_{1}<R_{1}, r_{2}<R_{2}$, satisfying

$$
\begin{aligned}
& A_{1} F_{1}\left(r_{1}, r_{2}\right)>r_{1}, \quad A_{2} F_{2}\left(r_{1}, r_{2}\right)>r_{2}, \\
& B_{1} F_{1}\left(R_{1}, 0\right)+C_{1} F_{1}\left(R_{1}, R_{2}\right)<R_{1}, \\
& B_{2} F_{2}\left(R_{1}, 0\right)+C_{2} F_{2}\left(R_{1}, R_{2}\right)<R_{2},
\end{aligned}
$$

then problem (1.1) at least has a positive solution.

Proof of Theorem 4.1 Let

$$
K_{r, R}:=\left\{x=\left(x_{1}, x_{2}\right) \in K: r_{i} \leq\left|x_{i}\right|_{i} \leq R_{i}\right\} \quad(i=1,2) .
$$

Then, for any $x(t) \in K_{r, R}$, we have

$$
x_{i}(t) \geq \delta(t)\left|x_{i}(t)\right|_{i}, \quad t \in[0, \xi],
$$

where $\delta(t)=\min \left\{\frac{t}{\xi}, \frac{\xi-t}{\xi}\right\}, t \in[0, \xi]$. 
On the one hand, for any $x(t) \in K_{r, R}$, we show that $T_{1}(x) \nless x_{1}$ if $\left|x_{1}\right|_{1}=r_{1}$ and $r_{2} \leq\left|x_{2}\right|_{2} \leq$ $R_{2}$. On the contrary, if $T_{1}(x)<x_{1}$, then we have

$$
\begin{aligned}
r_{1} & \geq \max _{t \in[0, \xi]} x_{1}(t) \geq \max _{t \in[0, \xi]} T_{1} x(t) \\
& =\max _{t \in[0, \xi]} \int_{0}^{1} G(t, s) h_{1}(s) f_{1}\left(x_{1}\left(s-\tau_{1}\right), x_{2}\left(s-\tau_{2}\right)\right) d s \\
& \geq \max _{t \in[0, \xi]} \int_{0}^{\sigma_{1}} G(t, s) h_{1}^{+}(s) f_{1}\left(x_{1}\left(s-\tau_{1}\right), x_{2}\left(s-\tau_{2}\right)\right) d s \\
& \geq \max _{t \in[0, \xi]} G(t, t) \cdot \int_{0}^{\sigma_{1}} G(s, s) h_{1}^{+}(s) f_{1}\left(x_{1}\left(s-\tau_{1}\right), x_{2}\left(s-\tau_{2}\right)\right) d s \\
& \geq k_{1} \max _{t \in[0, \xi]} G(t, t) \cdot \int_{\frac{\sigma_{1}+\tau_{2}}{2}}^{\sigma_{1}} G(s, s) h_{1}^{+}(s) F_{1}\left(x_{1}\left(s-\tau_{1}\right), x_{2}\left(s-\tau_{2}\right)\right) d s \\
& \geq k_{1} \max _{t \in[0, \xi]} G(t, t) \cdot \int_{\frac{\sigma_{1}+\tau_{2}}{2}}^{\sigma_{1}} G(s, s) h_{1}^{+}(s) F_{1}\left(\delta\left(s-\tau_{1}\right)\left|x_{1}\right|_{1}, \delta\left(s-\tau_{2}\right)\left|x_{2}\right|_{2}\right) d s \\
& \geq k_{1} \max _{t \in[0, \xi]} G(t, t) \cdot \int_{\frac{\sigma_{1}+\tau_{2}}{2}}^{\sigma_{1}} G(s, s) h_{1}^{+}(s) F_{1}\left(\gamma_{1}\left|x_{1}\right|_{1}, \gamma_{1}\left|x_{2}\right|_{2}\right) d s \\
& =k_{1} \gamma_{1}^{\alpha_{1}} \max _{t \in[0, \xi]} G(t, t) \cdot \int_{\frac{\sigma_{1}+\tau_{2}}{2}}^{\sigma_{1}} G(s, s) h_{1}^{+}(s) F_{1}\left(\left|x_{1}\right|_{1},\left|x_{2}\right|_{2}\right) d s \\
& \geq k_{1}, k_{1} \gamma_{1}^{\alpha_{1}} \max _{t \in[0, \xi]}^{\sigma_{1}} G(t, t) \cdot \int_{\frac{\sigma_{1}+\tau_{2}}{2}}^{\sigma_{1}} G(s, s) h_{1}^{+}(s) d s \cdot F_{1}\left(r_{1}, r_{2}\right) \\
& \geq k_{1} \gamma_{1}^{\alpha_{1}} \max _{t \in[0, \xi]} G(t, t) \cdot \int_{\frac{\sigma_{1}+\tau_{2}}{2}}^{\sigma_{1}} G(s, s) h_{1}^{+}(s) F_{1}\left(r_{1}, r_{2}\right) d s \\
&
\end{aligned}
$$

which implies a contradiction. In the similar way, for any $x(t) \in K_{r, R}$, we also can obtain $T_{2}(x) \nless x_{2}$ if $\left|x_{2}\right|_{2}=r_{2}$ and $r_{1} \leq\left|x_{1}\right|_{1} \leq R_{1}$.

On the one hand, for any $x(t) \in K_{r, R}$, we show that $T_{1}(x) \ngtr x_{1}$ if $\left|x_{1}\right|_{1}=R_{1}$ and $r_{2} \leq\left|x_{2}\right|_{2} \leq$ $R_{2}$. On the contrary, if $T_{1}(x)>x_{1}$, then we have

$$
\begin{aligned}
x_{1}(t)< & T_{1} x(t)=\int_{0}^{1} G(t, s) h_{1}(s) f_{1}\left(x_{1}\left(s-\tau_{1}\right), x_{2}\left(s-\tau_{2}\right)\right) d s \\
= & \int_{0}^{\xi} G(t, s) h_{1}^{+}(s) f_{1}\left(x_{1}\left(s-\tau_{1}\right), x_{2}\left(s-\tau_{2}\right)\right) d s \\
& -\int_{\xi}^{1} G(t, s) h_{1}^{-}(s) f_{1}\left(x_{1}\left(s-\tau_{1}\right), x_{2}\left(s-\tau_{2}\right)\right) d s \\
\leq & \int_{0}^{\xi} G(s, s) h_{1}^{+}(s) f_{1}\left(x_{1}\left(s-\tau_{1}\right), x_{2}\left(s-\tau_{2}\right)\right) d s \\
= & \left(\int_{0}^{\tau_{1}}+\int_{\tau_{1}}^{\tau_{2}}+\int_{\tau_{2}}^{\xi}\right) G(s, s) h_{1}^{+}(s) f_{1}\left(x_{1}\left(s-\tau_{1}\right), x_{2}\left(s-\tau_{2}\right)\right) d s \\
= & \int_{0}^{\tau_{1}} G(s, s) h_{1}^{+}(s) f_{1}(0,0) d s+\int_{\tau_{1}}^{\tau_{2}} G(s, s) h_{1}^{+}(s) f_{1}\left(x_{1}\left(s-\tau_{1}\right), 0\right) d s \\
& +\int_{\tau_{2}}^{\xi} G(s, s) h_{1}^{+}(s) f_{1}\left(x_{1}\left(s-\tau_{1}\right), x_{2}\left(s-\tau_{2}\right)\right) d s
\end{aligned}
$$




$$
\begin{aligned}
\leq & \int_{\tau_{1}}^{\tau_{2}} G(s, s) h_{1}^{+}(s) F_{1}\left(x_{1}\left(s-\tau_{1}\right), 0\right) d s \\
& +\int_{\tau_{2}}^{\xi} G(s, s) h_{1}^{+}(s) F_{1}\left(x_{1}\left(s-\tau_{1}\right), x_{2}\left(s-\tau_{2}\right)\right) d s \\
\leq & \int_{\tau_{1}}^{\tau_{2}} G(s, s) h_{1}^{+}(s) F_{1}\left(R_{1}, 0\right) d s+\int_{\tau_{2}}^{\xi} G(s, s) h_{1}^{+}(s) F_{1}\left(R_{1}, R_{2}\right) d s \\
< & R_{1},
\end{aligned}
$$

which implies a contradiction. In the similar way, for any $x(t) \in K_{r, R}$, we also can obtain $T_{2}(x) \ngtr x_{2}$ if $\left|x_{2}\right|_{2}=R_{2}$ and $r_{1} \leq\left|x_{1}\right|_{1} \leq R_{1}$.

Therefore, from (a) of Lemma 2.3, the operator $T$ has a fixed point $x$ in $K$ with $r_{i} \leq\left|x_{i}\right| \leq$ $R_{i}$.

As in a similar proof to Theorem 4.1, we also get the following.

Theorem 4.2 Assume that $\left(\mathrm{H}_{1}\right)-\left(\mathrm{H}_{4}\right)$ hold. If there exist four positive constants $r_{1}, r_{2}, R_{1}$, $R_{2}$ with $r_{1}<R_{1}, r_{2}<R_{2}$, satisfying

$$
\begin{aligned}
& A_{1} F_{1}\left(R_{1}, r_{2}\right)>R_{1}, \quad A_{2} F_{2}\left(r_{1}, R_{2}\right)>R_{2}, \\
& B_{1} F_{1}\left(r_{1}, 0\right)+C_{1} F_{1}\left(r_{1}, R_{2}\right)<r_{1}, \\
& B_{2} F_{2}\left(R_{1}, 0\right)+C_{2} F_{2}\left(R_{1}, r_{2}\right)<r_{2},
\end{aligned}
$$

then problem (1.1) at least has a positive solution.

\section{Examples}

Example 5.1 Now we consider the following problem:

$$
\left\{\begin{array}{l}
x_{1}^{\prime \prime}(t)+h_{1}(t) f_{1}\left(x_{1}\left(t-\tau_{1}\right), x_{2}\left(t-\tau_{2}\right)\right), \quad t>0, \\
x_{2}^{\prime \prime}(t)+h_{2}(t) f_{2}\left(x_{1}\left(t-\tau_{1}\right), x_{2}\left(t-\tau_{2}\right)\right), \quad t>0, \\
x_{1}(t)=0, \quad-\tau_{1} \leq t \leq 0, \quad \text { and } \quad x_{1}(1)=0, \\
x_{2}(t)=0, \quad-\tau_{2} \leq t \leq 0, \quad \text { and } \quad x_{2}(1)=0,
\end{array}\right.
$$

where $\tau_{1}=\frac{1}{10}, \tau_{2}=\frac{1}{5}$,

$$
\begin{aligned}
& h_{1}(t)=h_{2}(t)= \begin{cases}400\left(\frac{1}{2}-t\right), & t \in\left[0, \frac{1}{2}\right], \\
0, & t \in\left[\frac{1}{2}, \frac{7}{10}\right], \\
-\frac{5}{3} t+\frac{7}{6}, & t \in\left[\frac{7}{10}, 1\right]\end{cases} \\
& f_{1}=\frac{\left(x_{1}+x_{2}\right)^{\frac{1}{2}}}{\frac{3}{2}+\frac{1}{4} \sin ^{2}\left(x_{1}+x_{2}\right)}, \\
& f_{2}=\frac{\left(x_{1}+x_{2}\right)^{\frac{1}{3}}}{\frac{4}{3}+\frac{1}{3 \pi} \arctan \left(x_{1}^{2} x_{2}^{4}\right)} .
\end{aligned}
$$


It is obvious that $\left(\mathrm{H}_{1}\right)$ and $\left(\mathrm{H}_{2}\right)$ hold. Furthermore, we also have

$$
\begin{aligned}
& \frac{1}{2} F_{1}\left(x_{1}, x_{2}\right)=\frac{1}{2}\left(x_{1}+x_{2}\right)^{\frac{1}{2}} \leq f_{1} \leq\left(x_{1}+x_{2}\right)^{\frac{1}{2}}=F_{1}\left(x_{1}, x_{2}\right), \\
& \frac{1}{2} F_{2}\left(x_{1}, x_{2}\right)=\frac{1}{2}\left(x_{1}+x_{2}\right)^{\frac{1}{3}} \leq f_{2} \leq\left(x_{1}+x_{2}\right)^{\frac{1}{3}}=F_{2}\left(x_{1}, x_{2}\right),
\end{aligned}
$$

which shows that $\left(\mathrm{H}_{3}\right)$ holds. Now, we prove that $\left(\mathrm{H}_{4}\right)$ holds.

Firstly, we show that there exist $\sigma_{i}$ satisfying $\frac{1}{10}<\sigma_{i}<\frac{1}{5}$ such that

$$
\theta^{2} k_{1}\left(\sigma_{1}-\tau_{2}\right)^{\frac{1}{2}} \int_{\sigma_{1}}^{\xi} G(t, s) h_{1}^{+}(s) d s \geq \xi^{\frac{1}{2}} \int_{\xi+\tau_{2}}^{1} G(t, s) h_{1}^{-}(s) d s
$$

and

$$
\theta^{2} k_{2}\left(\sigma_{2}-\tau_{2}\right)^{\frac{1}{3}} \int_{\sigma_{2}}^{\xi} G(t, s) h_{2}^{+}(s) d s \geq \xi^{\frac{1}{3}} \int_{\xi+\tau_{2}}^{1} G(t, s) h_{2}^{-}(s) d s
$$

For fixed $\theta=\frac{1}{2}, \sigma_{1}=\sigma_{2}=\frac{2}{5}$, we set

$$
\begin{aligned}
& M(t)=\theta^{2} \frac{1}{2}\left(\sigma_{1}-\tau_{2}\right)^{\frac{1}{2}} \int_{\sigma_{1}}^{\xi} G(t, s) h_{1}^{+}(s) d s=\frac{50}{\sqrt{5}} \int_{\frac{2}{5}}^{\frac{1}{2}} G(t, s)\left(\frac{1}{2}-s\right) d s, \\
& N(t)=\xi^{\frac{1}{2}} \int_{\xi+\tau_{2}}^{1} G(t, s) h_{1}^{-}(s) d s=\frac{1}{\sqrt{2}} \int_{\frac{7}{10}}^{1} G(t, s)\left(\frac{5}{3} s-\frac{7}{6}\right) d s, \quad t \in[0,1] .
\end{aligned}
$$

For $t \in\left[0, \frac{2}{5}\right]$, we have

$$
\begin{aligned}
& M(t)=\frac{50 t}{\sqrt{5}} \int_{\frac{2}{5}}^{\frac{1}{2}}(1-s)\left(\frac{1}{2}-s\right) d s=\frac{17}{120 \sqrt{5}} t, \\
& N(t)=\frac{t}{\sqrt{2}} \int_{\frac{7}{10}}^{1}(1-s)\left(\frac{5}{3} s-\frac{7}{6}\right) d s=\frac{3}{400 \sqrt{2}} t .
\end{aligned}
$$

It is obvious that $M(t) \geq N(t), \forall t \in\left[0, \frac{2}{5}\right]$.

For $t \in\left[\frac{2}{5}, \frac{1}{2}\right]$, we have

$$
\begin{aligned}
M(t) & =\frac{50}{\sqrt{5}}\left[\int_{\frac{2}{5}}^{t}(1-t) s\left(\frac{1}{2}-s\right) d s+\int_{t}^{\frac{1}{2}} t(1-s)\left(\frac{1}{2}-s\right) d s\right] \\
& =\frac{50}{\sqrt{5}}\left(\frac{1}{6} t^{3}-\frac{1}{4} t^{2}+\frac{737}{6000} t-\frac{7}{375}\right), \\
N(t) & =\frac{t}{\sqrt{2}} \int_{\frac{7}{10}}^{1}(1-s)\left(\frac{5}{3} s-\frac{7}{6}\right) d s=\frac{3}{400 \sqrt{2}} t .
\end{aligned}
$$

Via some computations, we have

$$
M(t)_{\min }=M\left(\frac{1}{2}\right)=\frac{13}{240 \sqrt{5}}>\frac{3}{800 \sqrt{2}}=N\left(\frac{1}{2}\right)=N(t)_{\max }
$$

which implies that $M(t)>N(t), \forall t \in\left[\frac{2}{5}, \frac{1}{2}\right]$. 
For $t \in\left[\frac{1}{2}, \frac{7}{10}\right]$, we have

$$
\begin{aligned}
& M(t)=\frac{50(1-t)}{\sqrt{5}} \int_{\frac{2}{5}}^{\frac{1}{2}} s\left(\frac{1}{2}-s\right) d s=\frac{13}{120 \sqrt{5}}(1-t), \\
& N(t)=\frac{t}{\sqrt{2}} \int_{\frac{7}{10}}^{1}(1-s)\left(\frac{5}{3} s-\frac{7}{6}\right) d s=\frac{3}{400 \sqrt{2}} t .
\end{aligned}
$$

Via some computations, we have

$$
M(t)_{\min }=M\left(\frac{7}{10}\right)=\frac{13}{400 \sqrt{5}}>\frac{21}{4000 \sqrt{2}}=N\left(\frac{7}{10}\right)=N(t)_{\max },
$$

which implies that $M(t)>N(t), \forall t \in\left[\frac{1}{2}, \frac{7}{10}\right]$.

For $t \in\left[\frac{7}{10}, 1\right]$, we have

$$
\begin{aligned}
M(t) & =\frac{50(1-t)}{\sqrt{5}} \int_{\frac{2}{5}}^{\frac{1}{2}} s\left(\frac{1}{2}-s\right) d s=\frac{13}{120 \sqrt{5}}(1-t), \\
N(t) & =\frac{1}{\sqrt{2}}\left[\int_{\frac{7}{10}}^{t}(1-t) s\left(\frac{5}{3} s-\frac{7}{6}\right) d s+\int_{t}^{1} t(1-s)\left(\frac{5}{3} s-\frac{7}{6}\right) d s\right] \\
& =\frac{1}{\sqrt{2}}\left(-\frac{5}{18} t^{3}+\frac{7}{12} t^{2}-\frac{481}{1200} t+\frac{343}{3600}\right) .
\end{aligned}
$$

Denote $g(t)=M(t)-N(t)$ and $g(1)=0$. Since

$$
g^{\prime}(t)=\frac{5}{6 \sqrt{2}} t^{2}-\frac{7}{6 \sqrt{2}} t+\frac{481}{1200 \sqrt{2}}-\frac{13}{120 \sqrt{5}}<0, \quad t \in\left[\frac{7}{10}, 1\right],
$$

$g(t)$ is strictly decreasing on $\left[\frac{7}{10}, 1\right]$. Then $g(t) \geq 0, \forall t \in\left[\frac{7}{10}, 1\right]$, which implies that $M(t) \geq$ $N(t), \forall t \in\left[\frac{7}{10}, 1\right]$.

So from these discussions, we have $M(t) \geq N(t), \forall t \in[0,1]$, which means that (5.2) holds. Hence, in the similar way, we also see that the inequality (5.3) is true.

Secondly, let

$$
\begin{aligned}
\Gamma(\rho) & =\min \left\{A_{1} F_{1}(\rho, 0), A_{2} F_{2}(0, \rho)\right\}=\min \left\{A_{1} \rho^{\frac{1}{2}}, A_{2} \rho^{\frac{1}{3}}\right\}, \\
\Lambda(\rho) & =\max \left\{B_{1} F_{1}(\rho, 0)+C_{1} F_{1}(\rho, \rho), B_{2} F_{2}(\rho, 0)+C_{2} F_{2}(\rho, \rho)\right\} \\
& =\max \left\{B_{1} \rho^{\frac{1}{2}}+C_{1}(2 \rho)^{\frac{1}{2}}, B_{2} \rho^{\frac{1}{3}}+C_{2}(2 \rho)^{\frac{1}{3}}\right\} .
\end{aligned}
$$

It is obvious that there exist a sufficiently small constant $r>0$ and a sufficiently large constant $R>0$ such that

$$
\begin{aligned}
& \min \left\{A_{1} r^{\frac{1}{2}}, A_{2} r^{\frac{1}{3}}\right\}>r, \\
& \max \left\{B_{1} R^{\frac{1}{2}}+C_{1}(2 R)^{\frac{1}{2}}, B_{2} R^{\frac{1}{3}}+C_{2}(2 R)^{\frac{1}{3}}\right\}<R .
\end{aligned}
$$

Then, by Theorem 3.1, problem (5.1) has a positive solution. 
Example 5.2 Now we consider the following problem:

$$
\begin{cases}x_{1}^{\prime \prime}(t)+h_{1}(t) f_{1}\left(x_{1}\left(t-\tau_{1}\right), x_{2}\left(t-\tau_{2}\right)\right), & t>0 \\ x_{2}^{\prime \prime}(t)+h_{2}(t) f_{2}\left(x_{1}\left(t-\tau_{1}\right), x_{2}\left(t-\tau_{2}\right)\right), & t>0 \\ x_{1}(t)=0, \quad-\tau_{1} \leq t \leq 0, \quad \text { and } \quad x_{1}(1)=0 & \\ x_{2}(t)=0, \quad-\tau_{2} \leq t \leq 0, \quad \text { and } \quad x_{2}(1)=0\end{cases}
$$

where $\tau_{1}=\frac{1}{10}, \tau_{2}=\frac{1}{5}$,

$$
\begin{array}{ll}
h_{1}(t)=h_{2}(t)= \begin{cases}\frac{1}{2}-t, & t \in\left[0, \frac{1}{2}\right], \\
0, & t \in\left[\frac{1}{2}, \frac{7}{10}\right], \\
10^{-4}\left(-\frac{5}{3} t+\frac{7}{6}\right), & t \in\left[\frac{7}{10}, 1\right],\end{cases} \\
f_{1}=\frac{\left(x_{1}+x_{2}\right)^{2}}{1+\left|\sin \left(x_{1} x_{2}\right)\right|}, \\
f_{2}=\frac{\left(x_{1}+x_{2}\right)^{3}}{1+\left|\sin \left(x_{1} x_{2}\right)\right|} .
\end{array}
$$

It is obvious that $\left(\mathrm{H}_{1}\right)$ and $\left(\mathrm{H}_{2}\right)$ hold. Moreover, we have

$$
\begin{array}{ll}
\frac{1}{2} F_{1}\left(x_{1}, x_{2}\right)=\frac{1}{2}\left(x_{1}+x_{2}\right)^{2} \leq f_{1} \leq\left(x_{1}+x_{2}\right)^{2}=F_{1}\left(x_{1}, x_{2}\right), & k_{1}>0 \\
\frac{1}{2} F_{2}\left(x_{1}, x_{2}\right)=\frac{1}{2}\left(x_{1}+x_{2}\right)^{3} \leq f_{2} \leq\left(x_{1}+x_{2}\right)^{3}=F_{2}\left(x_{1}, x_{2}\right), & k_{2}>0
\end{array}
$$

which shows that $\left(\mathrm{H}_{3}\right)$ holds. Now, we prove that $\left(\mathrm{H}_{4}\right)$ holds.

Firstly, we show that there exist $\sigma_{i}$ satisfying $\frac{1}{10}<\sigma_{i}<\frac{1}{5}$ such that

$$
\theta^{2} k_{1}\left(\sigma_{1}-\tau_{2}\right)^{2} \int_{\sigma_{1}}^{\xi} G(t, s) h_{1}^{+}(s) d s \geq \xi^{2} \int_{\xi+\tau_{2}}^{1} G(t, s) h_{1}^{-}(s) d s
$$

and

$$
\theta^{2} k_{2}\left(\sigma_{2}-\tau_{2}\right)^{3} \int_{\sigma_{2}}^{\xi} G(t, s) h_{2}^{+}(s) d s \geq \xi^{3} \int_{\xi+\tau_{2}}^{1} G(t, s) h_{2}^{-}(s) d s
$$

For fixed $\theta=\frac{1}{2}, \sigma_{1}=\sigma_{2}=\frac{2}{5}$, we set

$$
\begin{aligned}
& m(t)=\theta^{2} \frac{1}{2}\left(\sigma_{1}-\tau_{2}\right)^{2} \int_{\sigma_{1}}^{\xi} G(t, s) h_{1}^{+}(s) d s=\frac{1}{200} \int_{\frac{2}{5}}^{\frac{1}{2}} G(t, s)\left(\frac{1}{2}-s\right) d s, \\
& n(t)=\xi^{2} \int_{\xi+\tau_{2}}^{1} G(t, s) h_{1}^{-}(s) d s=\frac{1}{4 \times 10^{4}} \int_{\frac{7}{10}}^{1} G(t, s)\left(\frac{5}{3} s-\frac{7}{6}\right) d s, \quad t \in[0,1] .
\end{aligned}
$$

For $t \in\left[0, \frac{2}{5}\right]$, we have

$$
m(t)=\frac{t}{200} \int_{\frac{2}{5}}^{\frac{1}{2}}(1-s)\left(\frac{1}{2}-s\right) d s=\frac{17}{12 \times 10^{5}} t
$$




$$
n(t)=\frac{t}{4 \times 10^{4}} \int_{\frac{7}{10}}^{1}(1-s)\left(\frac{5}{3} s-\frac{7}{6}\right) d s=\frac{3}{16 \times 10^{6}} t .
$$

It is obvious that $m(t) \geq n(t), \forall t \in\left[0, \frac{2}{5}\right]$.

For $t \in\left[\frac{2}{5}, \frac{1}{2}\right]$, we have

$$
\begin{aligned}
m(t) & =\frac{1}{200}\left[\int_{\frac{2}{5}}^{t}(1-t) s\left(\frac{1}{2}-s\right) d s+\int_{t}^{\frac{1}{2}} t(1-s)\left(\frac{1}{2}-s\right) d s\right] \\
& =\frac{1}{200}\left(\frac{1}{6} t^{3}-\frac{1}{4} t^{2}+\frac{737}{6000} t-\frac{7}{375}\right), \\
n(t) & =\frac{t}{4 \times 10^{4}} \int_{\frac{7}{10}}^{1}(1-s)\left(\frac{5}{3} s-\frac{7}{6}\right) d s=\frac{3}{16 \times 10^{6}} t .
\end{aligned}
$$

Via some computations, we have

$$
m(t)_{\min }=m\left(\frac{1}{2}\right)=\frac{13}{24 \times 10^{5}}>\frac{3}{32 \times 10^{6}}=n\left(\frac{1}{2}\right)=n(t)_{\max }
$$

which implies that $m(t)>n(t), \forall t \in\left[\frac{2}{5}, \frac{1}{2}\right]$.

For $t \in\left[\frac{1}{2}, \frac{7}{10}\right]$, we have

$$
\begin{aligned}
& m(t)=\frac{1-t}{200} \int_{\frac{2}{5}}^{\frac{1}{2}} s\left(\frac{1}{2}-s\right) d s=\frac{13}{12 \times 10^{5}}(1-t), \\
& n(t)=\frac{t}{4 \times 10^{4}} \int_{\frac{7}{10}}^{1}(1-s)\left(\frac{5}{3} s-\frac{7}{6}\right) d s=\frac{3}{16 \times 10^{6}} t .
\end{aligned}
$$

Via some computations, we have

$$
m(t)_{\min }=m\left(\frac{7}{10}\right)=\frac{13}{4 \times 10^{6}}>\frac{21}{16 \times 10^{7}}=n\left(\frac{7}{10}\right)=n(t)_{\max },
$$

which implies that $m(t)>n(t), \forall t \in\left[\frac{1}{2}, \frac{7}{10}\right]$.

For $t \in\left[\frac{7}{10}, 1\right]$, we have

$$
\begin{aligned}
m(t) & =\frac{1-t}{200} \int_{\frac{2}{5}}^{\frac{1}{2}} s\left(\frac{1}{2}-s\right) d s=\frac{13}{12 \times 10^{5}}(1-t), \\
n(t) & =\frac{1}{4 \times 10^{4}}\left[\int_{\frac{7}{10}}^{t}(1-t) s\left(\frac{5}{3} s-\frac{7}{6}\right) d s+\int_{t}^{1} t(1-s)\left(\frac{5}{3} s-\frac{7}{6}\right) d s\right] \\
& =\frac{1}{4 \times 10^{4}}\left(-\frac{5}{18} t^{3}+\frac{7}{12} t^{2}-\frac{481}{1200} t+\frac{343}{3600}\right) .
\end{aligned}
$$

Let $\omega(t)=m(t)-n(t)$ satisfying $\omega(1)=0$. Since

$$
\omega^{\prime}(t)=\frac{5}{24 \times 10^{4}} t^{2}-\frac{7}{24 \times 10^{4}} t-\frac{13}{16 \times 10^{6}}<0, \quad t \in\left[\frac{7}{10}, 1\right],
$$

it means that $\omega(t)$ is strictly decreasing on $\left[\frac{7}{10}, 1\right]$. Then $\omega(t) \geq 0, \forall t \in\left[\frac{7}{10}, 1\right]$, namely, $m(t) \geq n(t), \forall t \in\left[\frac{7}{10}, 1\right]$. 
So from these discussions, we have $m(t) \geq n(t), \forall t \in[0,1]$, which means that (5.5) holds. Hence, by a similar method, we also can see that the inequality (5.6) is true.

Secondly, let

$$
\begin{aligned}
\Gamma(\rho) & =\min \left\{A_{1} F_{1}(\rho, 0), A_{2} F_{2}(0, \rho)\right\}=\min \left\{A_{1} \rho^{2}, A_{2} \rho^{3}\right\}, \\
\Lambda(\rho) & =\max \left\{B_{1} F_{1}(\rho, 0)+C_{1} F_{1}(\rho, \rho), B_{2} F_{2}(\rho, 0)+C_{2} F_{2}(\rho, \rho)\right\} \\
& =\max \left\{B_{1} \rho^{2}+C_{1}(2 \rho)^{2}, B_{2} \rho^{3}+C_{2}(2 \rho)^{3}\right\} .
\end{aligned}
$$

It is obvious that there exist a sufficiently small constant $r>0$ and a sufficiently large constant $R>0$ such that

$$
\begin{aligned}
& \min \left\{A_{1} R^{2}, A_{2} R^{3}\right\}>R, \\
& \max \left\{B_{1} r^{2}+C_{1}(2 r)^{2}, B_{2} r^{3}+C_{2}(2 r)^{3}\right\}<r .
\end{aligned}
$$

Then, by Theorem 3.2, problem (5.4) has a positive solution.

Example 5.3 Now we consider the following problem:

$$
\begin{cases}x_{1}^{\prime \prime}(t)+h_{1}(t) f_{1}\left(x_{1}\left(t-\tau_{1}\right), x_{2}\left(t-\tau_{2}\right)\right), & t>0 \\ x_{2}^{\prime \prime}(t)+h_{2}(t) f_{2}\left(x_{1}\left(t-\tau_{1}\right), x_{2}\left(t-\tau_{2}\right)\right), & t>0 \\ x_{1}(t)=0, \quad-\tau_{1} \leq t \leq 0, \quad \text { and } \quad x_{1}(1)=0 & \\ x_{2}(t)=0, \quad-\tau_{2} \leq t \leq 0, \quad \text { and } \quad x_{2}(1)=0\end{cases}
$$

where $\tau_{1}=\frac{1}{10}, \tau_{2}=\frac{1}{5}$,

$$
\begin{aligned}
& h_{1}(t)=h_{2}(t)= \begin{cases}400\left(\frac{1}{2}-t\right), & t \in\left[0, \frac{1}{2}\right], \\
0, & t \in\left[\frac{1}{2}, \frac{7}{10}\right], \\
-\frac{5}{3} t+\frac{7}{6}, & t \in\left[\frac{7}{10}, 1\right],\end{cases} \\
& f_{1}=\left(x_{1}+x_{2}\right)^{\frac{1}{2},} \quad f_{2}=\left(x_{1}+x_{2}\right)^{\frac{1}{3}} .
\end{aligned}
$$

Choosing $\theta=\frac{1}{2}, k_{1}=k_{2}=\frac{1}{2}, \sigma_{1}=\sigma_{2}=\frac{2}{5}$. Then, from Example 5.1, it is obvious that $\left(\mathrm{H}_{1}\right)-$ $\left(\mathrm{H}_{4}\right)$ hold.

Now, we show that there exist $0<r_{i}<R_{i}$ such that

$$
\begin{aligned}
& k_{1} \gamma_{1}^{\frac{1}{2}} \max _{t \in\left[0, \frac{1}{2}\right]} G(t, t) \cdot \int_{\frac{\sigma_{1}+\frac{1}{5}}{2}}^{\sigma_{1}} G(s, s) h_{1}^{+}(s) d s \cdot\left(r_{1}+r_{2}\right)^{\frac{1}{2}}>r_{1}, \\
& k_{2} \gamma_{2}^{\frac{1}{3}} \max _{t \in\left[0, \frac{1}{2}\right]} G(t, t) \cdot \int_{\frac{\sigma_{2}+\frac{1}{5}}{2}}^{\sigma_{2}} G(s, s) h_{2}^{+}(s) d s \cdot\left(r_{1}+r_{2}\right)^{\frac{1}{3}}>r_{2}, \\
& \int_{\frac{1}{10}}^{\frac{1}{5}} G(s, s) h_{1}^{+}(s) d s \cdot R_{1}^{\frac{1}{2}}+\int_{\frac{1}{5}}^{\frac{1}{2}} G(s, s) h_{1}^{+}(s) d s \cdot\left(R_{1}+R_{2}\right)^{\frac{1}{2}}<R_{1}, \\
& \int_{\frac{1}{10}}^{\frac{1}{5}} G(s, s) h_{2}^{+}(s) d s \cdot R_{1}^{\frac{1}{3}}+\int_{\frac{1}{5}}^{\frac{1}{2}} G(s, s) h_{2}^{+}(s) d s \cdot\left(R_{1}+R_{2}\right)^{\frac{1}{3}}<R_{2} .
\end{aligned}
$$


Choosing $r_{1}=0.001, r_{2}=0.01, R_{1}=70, R_{2}=140$, we obtain

$$
\begin{aligned}
& \frac{50}{\sqrt{5}} \int_{\frac{3}{10}}^{\frac{2}{5}} s(1-s)\left(\frac{1}{2}-s\right) d s \cdot(0.001+0.01)^{\frac{1}{2}}=\frac{0.16875}{\sqrt{5}} \sqrt{0.011}>0.001, \\
& \frac{50}{\sqrt[3]{5}} \int_{\frac{3}{10}}^{\frac{2}{5}} s(1-s)\left(\frac{1}{2}-s\right) d s \cdot(0.001+0.01)^{\frac{1}{3}}=\frac{0.16875}{\sqrt[3]{5}} \sqrt[3]{0.011}>0.01 \\
& 400\left[\int_{\frac{1}{10}}^{\frac{1}{5}} s(1-s)\left(\frac{1}{2}-s\right) d s \cdot 70^{\frac{1}{2}}+\int_{\frac{1}{5}}^{\frac{1}{2}} s(1-s)\left(\frac{1}{2}-s\right) d s \cdot(70+140)^{\frac{1}{2}}\right] \\
& \quad=1.75 \sqrt{70}+3.69 \sqrt{210}<70, \\
& 400\left[\int_{\frac{1}{10}}^{\frac{1}{5}} s(1-s)\left(\frac{1}{2}-s\right) d s \cdot 70^{\frac{1}{3}}+\int_{\frac{1}{5}}^{\frac{1}{2}} s(1-s)\left(\frac{1}{2}-s\right) d s \cdot(70+140)^{\frac{1}{3}}\right] \\
& \quad=1.75 \sqrt[3]{70}+3.69 \sqrt[3]{210}<140,
\end{aligned}
$$

which implies that the inequalities (5.8)-(5.11) are true.

Therefore, from Theorem 4.1, one concludes that problem (5.7) has a positive solution.

\section{Acknowledgements}

We would like to thank the referee for his/her valuable comments and helpful suggestions which have led to an improvement of the presentation of this paper.

\section{Funding}

The authors were supported by the Fundamental Research Funds for the Central Universities (B200202003).

\section{Availability of data and materials}

Not applicable.

\section{Competing interests}

The authors declare that they have no competing interests.

\section{Authors' contributions}

All the authors contributed equally and significantly in writing this article. All the authors read and approved the final manuscript.

\section{Author details}

'Department of Mathematics, College of Science, Hohai University, 210098 Nanjing, P.R. China. ${ }^{2}$ Kunshan No. 2 Middle School, 235300 Kunshan, P.R. China.

\section{Publisher's Note}

Springer Nature remains neutral with regard to jurisdictional claims in published maps and institutional affiliations.

\section{Received: 9 November 2020 Accepted: 1 September 2021 Published online: 23 November 2021}

\section{References}

1. Bai, D., Xu, Y.: Positive solutions of second-order two-delay differential systems with twin-parameter. Nonlinear Anal. 63, 601-617 (2005)

2. Bai, D., Xu, Y.: Positive solutions and eigenvalue regions of two-delay singular systems with a twin parameter. Nonlinear Anal. 66, 2547-2564 (2007)

3. Cac, N.P., Fink, A.M., Gatica, J.A.: Nonnegative solutions of the radial Laplacian with nonlinearity that changes sign. Proc. Am. Math. Soc. 123, 1393-1398 (1995)

4. Candan, T: Existence of positive periodic solutions of first order neutral differential equations with variable coefficients. Appl. Math. Lett. 52, 142-148 (2016)

5. Candan, T.: Existence of positive periodic solution of second-order neutral differential equations. Turk. J. Math. 42, 797-806 (2018)

6. Carvalho, L.A.V., Ladeira, L.A.C., Martelli, M.: Forbidden periods in delay differential equations. Port. Math. 57, 259-271 (2000)

7. Dai, G., Han, X.: Global bifurcation and nodal solutions for fourth-order problems with sign-changing weight. Appl. Math. Comput. 219, 9399-9407 (2013) 
8. Guo, D., Lakshmikantham, V.: Nonlinear Problems in Abstract Cones. Academic Press, New York (1988)

9. Hale, J.K., Huang, W.: Global geometry of stable regions for two delay differential equations. J. Math. Anal. Appl. 178 344-362 (1993)

10. Lee, J.W., O'Regan, D.: Existence results for differential delay equations—II. Nonlinear Anal. 17, 683-702 (1991)

11. Lee, J.W., O'Regan, D.: Existence results for differential delay equations-I. J. Differ. Equ. 102, 342-359 (1993)

12. Liu, Y.: Boundary value problems on half-line for functional differential equations with infinite delay in a Banach space. Nonlinear Anal. 52, 1695-1708 (2003)

13. Ma, R., Gao, C., Han, X.: On linear and nonlinear fourth-order eigenvalue problems with indefinite weight. Nonlinear Anal. 74, 6965-6969 (2011)

14. Ma, R., Han, X.: Existence and multiplicity of positive solutions of a nonlinear eigenvalue problem with indefinite weight function. Appl. Math. Comput. 215, 1077-1083 (2009)

15. Precup, R.: A vector version of Krasnosel'skii's fixed point theorem in cones and poisitve periodic solutions of nonlinear systems. J. Fixed Point Theory Appl. 2, 141-151 (2007)

16. Tyagi, J.: Existence of nonnegative solutions for a class of semilinear elliptic systems with indefinite weight. Nonlinear Anal. 73, 2882-2889 (2010)

17. Yan, D., Pan, Z.: Positive solutions of nonlinear operator equations with sign-changing kernel and its applications. Appl. Math. Comput. 230, 675-679 (2014)

18. Yao, Q.: Existence and multiplicity of positive radial solutions for a semilinear elliptic equation with change of sign. Appl. Anal. 80, 65-77 (2001)

\section{Submit your manuscript to a SpringerOpen ${ }^{\circ}$ journal and benefit from:}

- Convenient online submission

- Rigorous peer review

- Open access: articles freely available online

- High visibility within the field

- Retaining the copyright to your article

Submit your next manuscript at $\mathbf{s p r i n g e r o p e n . c o m ~}$ 